\title{
ORIENTATION-REVERSING MORSE-SMALE DIFFEOMORPHISMS ON THE TORUS
}

\author{
BY \\ STEVE BATTERSON ${ }^{1}$
}

\begin{abstract}
For orientation-reversing diffeomorphisms on the torus necessary and sufficient conditions are given for an isotopy class to admit a Morse-Smale diffeomorphism with a specified periodic behavior.
\end{abstract}

A diffeomorphism is Morse-Smale provided it is structurally stable and has a finite nonwandering set [P-S]. Several recent papers have explored the relationship between the topology of these maps and their dynamics. In [F] Franks showed that the periodic behavior of a Morse-Smale diffeomorphism was restricted by its homology zeta function. For the homotopy class of the identity on a compact surface Narasimhan proved that virtually any periodic behavior consistent with the homology zeta function does indeed occur [N].

For orientation-reversing maps there are obstructions other than the homology zeta function. Blanchard and Franks [B-F] have shown that if an orientation-reversing homeomorphism of $S^{2}$ has periodic orbits which include two distinct odd periods, then the entropy of that map is positive. This implies that no orientationreversing Morse-Smale diffeomorphism on $S^{2}$ can have distinct odd periods. The following theorem was conjectured in [B-F] and has been proven by Handel.

THEOREM [H]. If $f: M^{2} \rightarrow M^{2}$ is an orientation-reversing homeomorphism of a compact oriented surface of genus $g$, and if $f$ has orbits with $g+2$ distinct odd periods, then the entropy of $f$ is positive.

Thus an orientation-reversing Morse-Smale diffeomorphism on the torus has orbits with at most two different odd periods. In this paper we investigate whether there are any further restrictions on its periodic behavior. In [B] we showed that it suffices to consider the isotopy classes of the toral diffeomorphisms induced by $\left(\begin{array}{lr}1 & 9 \\ 0 & -1\end{array}\right)$ and $\left(\begin{array}{ll}1 & 1 \\ 0 & -1\end{array}\right)$. We will show that there is a further obstruction in the former class but not in the latter.

I would like to thank John Franks and Lynn Narasimhan for their contributions to this paper.

Preliminaries. In this section we state our main result following some necessary definitions and background. We assume the reader is familiar with various standard terms and notation from dynamical systems. Further details are available in [B], [N] and [Ni].

Received by the editors November 26, 1979 and, in revised form, February 25, 1980.

AMS (MOS) subject classifications (1970). Primary 58F09, 58F20.

${ }^{1}$ Research supported in part by Emory University Summer Research Grant. 
Definition. The homology zeta function $\eta_{f}$ of a diffeomorphism $f$ is defined by $\eta_{f}(t)=\Pi_{k=0}^{\operatorname{dim} M} \operatorname{det}\left(I-f_{* k} t\right)^{(-1)^{k+1}}$ where $f_{* k}: H_{k}(M, R) \rightarrow H_{k}(M, R)$ is the induced map on $k$ th homology.

Let $\gamma$ be an orbit of period $p$ for a Morse-Smale diffeomorphism. The index of $\gamma$ is the dimension of $E_{x}^{u}$ for $x \in \gamma$. The orientation type $\Delta$ of $\gamma$ is defined to be +1 if $D f_{x}^{p}: E_{x}^{u} \rightarrow E_{x}^{u}$ preserves orientation. For a sink orbit define $\Delta=+1$. Otherwise $\Delta=-1$. We correspond a triple $(p, u, \Delta)$ to $\gamma$ which consists of the period, index, and orientation type of $\gamma$. For a diffeomorphism whose nonwandering set consists of a finite number of hyperbolic periodic points, its periodic data is defined to be the collection consisting of these triples (where the same triple can occur more than once provided it corresponds to different orbits).

The following theorem of Franks relates the homology zeta function to the periodic data.

TheOREM [F]. Let $f: M \rightarrow M$ be a $C^{1}$ map with a finite number of periodic orbits all of which are hyperbolic. Then $\eta_{f}(t)=\Pi_{i}\left(1-\Delta_{i} t^{p_{i}}\right)^{(-1)^{\mu_{i}+1}}$.

This theorem restricts the Morse-Smale periodic data that can occur in a given isotopy class. For orientation-preserving diffeomorphisms on $T^{2}$ there are only two other restrictions, each of which is obviously necessary.

TheOREM [B]. Let $\mathrm{g} \in \operatorname{Diff}\left(T^{2}\right)$ and suppose $\mathrm{g}$ preserves orientation. There exists a Morse-Smale diffeomorphism $f$ on $T^{2}$ isotopic to $g$ and with periodic data $\left\{\left(p_{i}, u_{i}, \Delta_{i}\right)\right\}_{i=1}^{n}$ if and only if

(1) $u_{i}=0$ and $u_{j}=2$ for some $i$ and $j$,

(2) $\eta_{g}(t)=\Pi_{i=1}^{n}\left(1-\Delta_{i} t^{p_{i}}\right)^{(-1)^{u_{i}+1}}$,

(3) $\Delta_{i}=+1$ for each $i$ with $u_{i} \in\{0,2\}$.

In this paper we investigate the situation for orientation-reversing diffeomorphisms. In both cases the work is simplified by the following two lemmas from [B].

Lemma 1. Let $A \in G l(2, Z)$ and suppose that both eigenvalues of $A$ are roots of unity. Then $A$ is similar over the integers to exactly one of the following matrices:

$$
\begin{gathered}
\left(\begin{array}{cc}
1 & m \\
0 & 1
\end{array}\right),\left(\begin{array}{rr}
-1 & m \\
0 & -1
\end{array}\right),\left(\begin{array}{rr}
0 & 1 \\
-1 & 0
\end{array}\right),\left(\begin{array}{rr}
0 & 1 \\
-1 & -1
\end{array}\right), \\
\left(\begin{array}{rr}
0 & -1 \\
1 & 1
\end{array}\right),\left(\begin{array}{rr}
1 & 0 \\
0 & -1
\end{array}\right),\left(\begin{array}{rr}
1 & 1 \\
0 & -1
\end{array}\right),
\end{gathered}
$$

$m \in\{0,1,2, \ldots\}$.

Lemma 2. Let $f, g, h \in \operatorname{Diff}(M)$ with $f$ Morse-Smale and $h f=g h$. Then $g$ is Morse-Smale and the periodic data of $g$ is equal to the periodic data of $f$.

The isotopy class of a diffeomorphism of the torus is completely determined by its action on first homology. Franks' theorem implies that if the diffeomorphism is Morse-Smale then the eigenvalues of the induced map are roots of unity. Thus to study the periodic behavior of Morse-Smale orientation-reversing diffeomorphisms of the torus, it suffices to consider the isotopy classes of maps on $T^{2}$ induced by 
$\left(\begin{array}{rr}1 & 9 \\ 0 & -1\end{array}\right)$ and $\left(\begin{array}{rr}1 & 1 \\ 0 & -1\end{array}\right)$. For example any periodic behavior that can be produced in the isotopy class determined by $\left(\begin{array}{ll}1 & 1 \\ 0 & -1\end{array}\right)$ can also be produced in $\left(\begin{array}{ll}0 & 1 \\ 1 & 0\end{array}\right)$ by simply applying the differentiable conjugacy induced on $T^{2} / Z^{2}$ by $\left(\begin{array}{rr}-1 & -1\end{array}\right)$.

We now partition the relevant isotopy classes into two groups. Those whose induced map on first homology is similar over the integers to $\left(\begin{array}{ll}1 & 0 \\ 0 & -1\end{array}\right)$ will be said to be of even type (because this group includes all those of the form $\left.\left(\begin{array}{ll}1 & 2 n \\ 0 & -1\end{array}\right)\right)$. Those sharing this relationship with $\left(\begin{array}{ll}1 & 1 \\ 0 & -1\end{array}\right)$ are of odd type.

Note that maps of both types have homology zeta function 1. Consequently this important invariant fails to distinguish periodic data that might occur in one class but not the other. The following definition characterizes that data in the present situation.

Definition. If $p$ is odd then the collection $\{(p, 0,1),(p, 2,-1),(2 p, 1,+1)\}$ is a minimal data collection.

Minimal data collections contain the fewest number of periodic orbits possible in a Morse-Smale diffeomorphism of the torus. We now can state the main result of this paper.

Theorem. Let $g \in \operatorname{Diff}\left(T^{2}\right)$ and suppose $g$ reverses orientation. There exists a Morse-Smale diffeomorphism $f$ on $T^{2}$ isotopic to $g$ and with periodic data $\left\{\left(p_{i}, u_{i}, \Delta_{i}\right)\right\}_{i=1}^{n}$ if and only if

(1) $u_{i}=0$ and $u_{j}=2$ for some $i$ and $j$,

(2) $\eta_{g}(t)=1=\prod_{i=1}^{n}\left(1-\Delta_{i} t^{p_{i}}\right)^{(-1)^{u_{i}+1}}$,

(3) $\Delta_{i}=+1$ if $u_{i}=0$ and $\Delta_{i}=(-1)^{p_{i}}$ if $u_{i}=2$,

(4) $\left\{p_{i}\right\}_{i=1}^{n}$ contains at most two different odd numbers,

(5) if $g$ is of even type then $\left\{\left(p_{i}, u_{i}, \Delta_{i}\right)\right\}_{i=1}^{n}$ is not a minimal data collection.

The necessity of conditions (1) and (3) is clear. The necessity of conditions (2) and (4) is a consequence of the results stated from [F] and [H] along with Lemma 1. In the following section we will show that conditions (1)-(4) are together sufficient for nonminimal data collections. In the final section we will show that minimal data collections can be realized by Morse-Smale diffeomorphisms in classes of odd type but not in classes of even type.

Nonminimal data collections. Suppose $P=\left\{\left(p_{i}, u_{i}, \Delta_{i}\right)\right\}_{i=1}^{n}$ satisfies (1)-(4) and is not minimal. We now outline the procedure involved in constructing Morse-Smale diffeomorphisms with periodic data $P$. First the data is organized into pieces and the torus is divided into cylinders (see Figure 1). Isotopies are then performed on each cylinder (annulus) so as to obtain a map which is isotopic to reflection across a vertical plane through the center of the torus. The isotopies put all the orbits with odd period $r$ in the top cylinder. Odd period $q$ orbits are put on the bottom cylinder. Some of the even orbits will also be put into these cylinders with each remaining orbit isotoped into a pair of side cylinders of the same height.

Employing Lemma 2, we will have completed the argument for nonminimal data in classes of even type. Finally adding another cylinder on each side, putting a Dehn twist in exactly one of them, and then flipping, we can preserve the periodic data but change the isotopy class to one of odd type. 


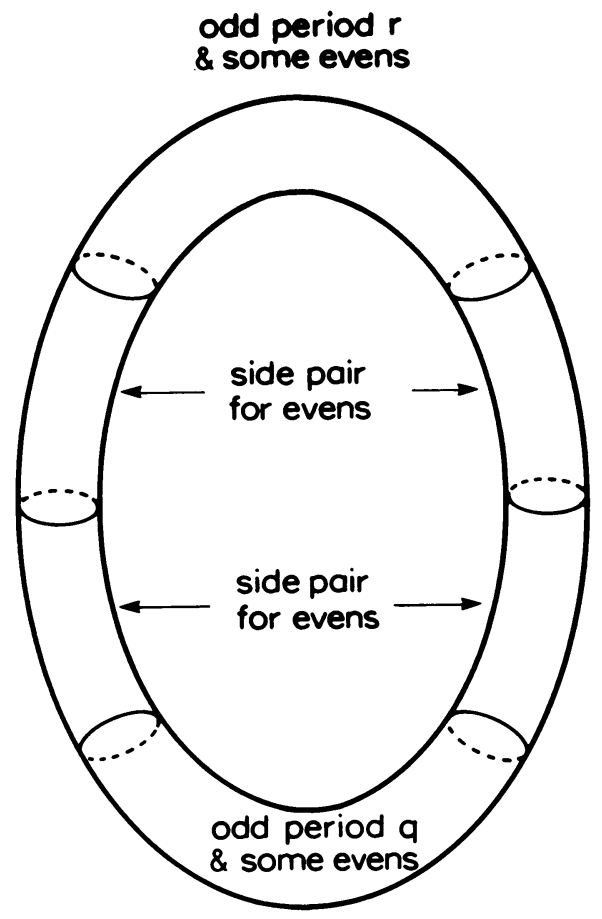

FIGURE 1

To begin the construction we algebraically organize the data $P$ into pieces. The data in each piece is sufficiently compatible that it can be realized on annulus or pair of annuli. Applying Lemma 5 of [B] and Lemma 2.1 of [N] the data can be put into the following form where $q$ and $r$ are odd and each $s_{i}$ is even.

$$
\begin{aligned}
& \prod_{i=1}^{n}\left(1-\Delta_{i} t^{p_{i}}\right)^{(-1)^{u_{i+1}}}=\left[\frac{1-t^{q}}{1-t^{q}}\right]^{m_{1}}\left[\frac{1+t^{q}}{1+t^{q}}\right]^{m_{2}}\left[\frac{\left(1-t^{2 q}\right)}{\left(1-t^{q}\right)\left(1+t^{q}\right)}\right]^{m_{3}} \\
& \text { - } \prod_{i=1}^{m_{4}} \frac{\left(1-t^{q}\right)\left(1+t^{q}\right)\left(1+t^{2 q}\right) \ldots\left(1+t^{2^{a_{i}-1} q}\right)}{1-t^{2^{a_{i} q}}} \\
& \cdot\left[\frac{1-t^{r}}{1-t^{r}}\right]^{m_{5}}\left[\frac{1+t^{r}}{1+t^{r}}\right]^{m_{6}}\left[\frac{\left(1-t^{2 r}\right)}{\left(1-t^{r}\right)\left(1+t^{r}\right)}\right]^{m_{7}} \\
& \cdot \prod_{i=1}^{m_{8}} \frac{\left(1-t^{r}\right)\left(1+t^{r}\right)\left(1+t^{2 r}\right) \ldots\left(1+t^{2^{a_{i}-1} r}\right)}{1-t^{2^{a_{r}}}} \\
& \cdot \prod_{i=1}^{m_{9}} \frac{\left(1-t^{s_{i}}\right)\left(1+t^{s_{i}}\right)\left(1+t^{2 s_{i}}\right) \ldots\left(1+t^{2^{a_{i}-s_{s}}}\right)}{\left(1-t^{2^{a_{i}}}\right)}
\end{aligned}
$$

Let $A=\left\{x \in \mathbf{R}^{2}|1<| x \mid<3\right\}, C=\{x \in A|| x \mid=2\}$ and $h$ be the diffeomorphism of $A$ onto itself obtained by reflecting about $C$. Suppose $m_{1}+m_{2}+m_{3}+$ $m_{4}>0$ and that the data $Q$ generated by these terms includes at least one sink. We 
must now construct an orientation-reversing diffeomorphism of $A$ into itself in which $C$ is an invariant set containing all of the orbits of period $q$. The periodic data of this map will be $Q$.

EXAMPLE. $m_{1}=m_{2}=m_{3}=1, m_{4}=0, q=3$.

The desired map is obtained by composing the following three.

(1) The time-one map of a flow whose dynamics are shown in Figure 2.

(2) Rotation by $2 \pi / 3$.

(3) $h$.

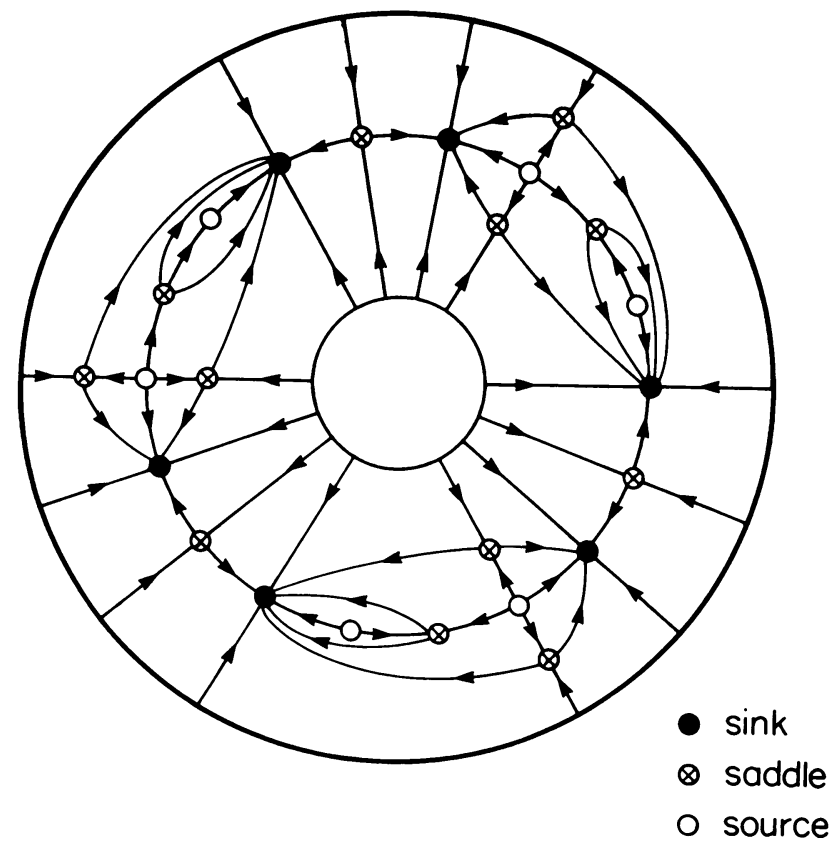

FIGURE 2

Note that the rotation near the boundary can be removed by a further isotopy.

LEMMA 3. For any $n>0$, there exists an orientation-reversing diffeomorphism $f$ of $D=\left\{x \in \mathbf{R}^{2}|| x \mid<1\right\}$ onto its image with periodic data

$$
\left\{\left(2^{n+1}, 0,1\right),(1,1,-1),(2,1,-1),\left(2^{2}, 1,-1\right), \ldots,\left(2^{n}, 1,-1\right)\right\} .
$$

Furthermore, for some $R>0, \varepsilon>0$, the restriction of $f$ to the annulus $\left\{x \in \mathbf{R}^{2}|1-R<| x \mid<1\right\}$ is given by $f(r, \theta)=(r-\varepsilon, \pi-\theta)$ and there exists a filtration of $D$ for $f$.

PRoof. In the proof of Lemma 3.1 in [N] define $h_{0}(r, \theta)=(r, \pi-\theta)$.

LEMMA 4. If $Q$ is nonempty and contains a sink then there exists an orientation-reversing diffeomorphism $f$ of $A$ into itself with periodic data $Q$. Furthermore, for some $R>0, \varepsilon>0$ any point $x$ within $R$ of either boundary is mapped along radii to the point between $x$ and $h(x)$ whose distance is $\varepsilon$ from $h(x)$. There is also a filtration of $A$ for $f$. 
Proof. The construction of $f$ involves combining the techniques of the example with Lemma 3.

Now suppose $m_{5}+m_{6}+m_{7}+m_{8}>0$ and the corresponding data includes a source. Then using the inverse of the Lemma 4 construction yields an overflowing invariant orientation-reversing diffeomorphism of $A$. The periodic data of this map includes all of the period $r$ data of $P$.

Referring back to Figure 1, we have the diffeomorphism defined on the top and bottom cylinders. Points near the boundaries of these cylinders are mapped over to the opposite arm and downward.

The diffeomorphism is completed by realizing the remaining even periodic data in pairs of cylinders along the sides. This is accomplished in Lemma 5 which involves a straightforward modification of Lemma 3.3 in [N]. Lemma 6 (see 3.2 of [N]) will be required if either there are no sinks or no sources in the $m_{1}$ through $m_{8}$ blocks.

Define $A_{b, c}^{i}=\left\{x \in R^{2}|b \leqslant| x \mid \leqslant c\right\}$ and let $Q$ be the disjoint union of the annuli $A_{1,3}^{1}$ and $A_{1,3}^{2}$.

LEMMA 5. For any even $s \geqslant 2, m \geqslant 0$ there exists a diffeomorphism $f$ of $\mathbb{Q}$ onto its image with periodic data

$$
\left\{\left(2^{m} s, 0,+1\right),(s, 1,1),(s, 1,-1),(2 s, 1,-1), \ldots,\left(2^{m-1} s, 1,-1\right)\right\} .
$$

Furthermore for some $R>0, \varepsilon>0, f$ maps the annulus $A_{1,1+R}^{i}$ along corresponding radii a distance $\varepsilon$ onto $A_{1-\varepsilon, 1+R-\varepsilon}^{j}$ and maps $A_{3-R, 3}^{i}$ onto $A_{3-R-\varepsilon, 3-\varepsilon}^{j}$ where $i \neq j$.

LEMMA 6. For any even $s \geqslant 2, m \geqslant 0$, there exists a diffeomorphism $f$ of $\mathbb{Q}$ onto its image which maps $A_{1,3}^{1}$ into $A_{1,3}^{2}, A_{1,3}^{2}$ into $A_{1,3}^{1}$, and has periodic data

$$
\left\{\left(2^{m} s, 0,+1\right),(s, 1,+1),(s, 1,-1),(2 s, 1,-1), \ldots,\left(2^{m-1} s, 1,-1\right)\right\} .
$$

Furthermore for some $R>0, \varepsilon>0, f$ maps $A_{1,1+R}^{i}$ and $A_{3-R, 3}^{i}$ along corresponding radii a distance $\varepsilon$ into $A_{1,3}^{j}$ where $i \neq j$.

Note that the nonminimality assumption implies $\sum m_{i} \geqslant 2$. Thus there will be at least two annuli to join together. If $\boldsymbol{P}$ has less than two odd periods, the construction is similar and involves either putting only even periods on the top and/or bottom annulus or putting the same odd periods on these annuli.

Minimal data collections. Let $\boldsymbol{P}$ be a minimal data collection (i.e., $\boldsymbol{P}=$ $\{(p, 0,+1),(p, 2,-1),(2 p, 1,+1)\}$ where $p$ is odd $)$. To complete the proof of the theorem we must show that $P$ can be realized in an isotopy class of odd type but not in one of even type. For $p=1$ the former is easy and the latter has been shown in an unpublished argument of Franks.

Suppose that $f$ is a Morse-Smale diffeomorphism on $T^{2}$ with periodic data $P$. Let $\left[q_{1}, q_{2}, \ldots, q_{2 p}\right],\left[r_{1}, r_{2}, \ldots, r_{p}\right]$, and $\left[s_{1}, s_{2}, \ldots, s_{p}\right]$ be the saddle, source, and sink orbits respectively.

Since there is only one saddle orbit, $f$ has no points which are heteroclinic between saddles. Consequently the unstable manifolds yield a cell decomposition of $T^{2}$. We obtain a graph $G^{u}$ from the 1 -skeleton as follows. $G^{u}$ has $3 p$ vertices 
corresponding to the sinks and saddles. Two vertices are adjacent if and only if their stable and unstable manifolds intersect. The valence of a vertex is the number of edges connected to it. From the stable manifold cell decomposition a graph, $G^{s}$, is similarly defined with source and saddle vertices. Let $G$ be the union of $G^{u}$ and $G^{s}$.

Due to the periodicity there are two possibilities for $G^{u}$ (see Figure 3). Either each saddle vertex is adjacent to one sink vertex or to two sink vertices. This is also the case for $G^{s}$. Since the graph $G$ must be connected, either $G^{u}$ or $G^{s}$ must also be connected. Thus we can assume that the saddles in $G^{u}$ are of valence two.
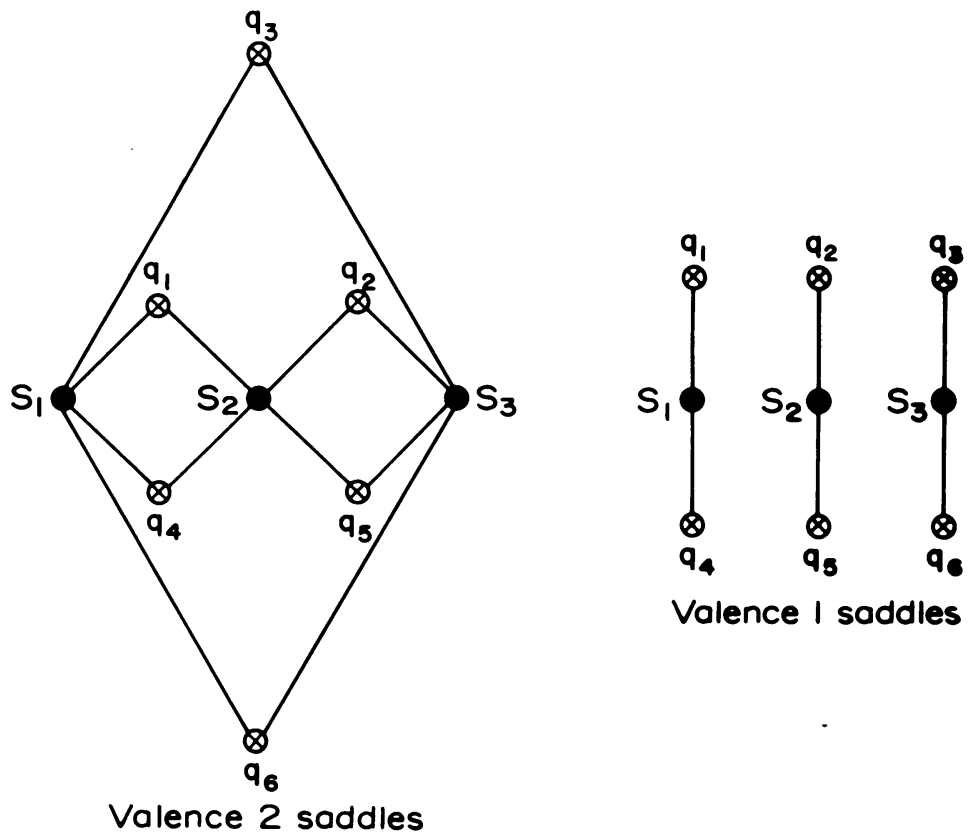

FIGURE 3 (possible $G^{u}$ for $p=3$ )

We wish to show that $G^{s}$ must also be a graph in which each saddle is of valence two. Suppose this is not the case and consider the stable manifold cell decomposition. The boundaries of the two cells are obtained from $G$ via the fact from $[P]$ that $\operatorname{cl}\left[W^{s}(s)\right] \cap W^{s}(q) \neq \varnothing$ if and only if $W^{s}(s) \cap W^{u}(q) \neq \varnothing$. If the saddles in $G^{s}$ were of valence one then this boundary would be disconnected. Thus we conclude that the graphs $G^{u}, G^{s}$, and $G$ are completely determined.

Following a procedure in [M] one could now compute $\pi\left(T^{2}, s_{1}\right)$, the fundamental group of $T^{2}$ at the basepoint $s_{1}$, from these graphs by first finding the free group $\pi\left(G^{u}, s_{1}\right)$ and then obtaining, from $G$, relations among the generators of $\pi\left(G^{u}, s_{1}\right)$. The action of $f$ on these generators is completely determined. Following a rather lengthy computation it could thus be shown that $f$ must be of odd type. We can avoid this computation by noting that $f$ can only be of one type and then showing that maps of odd type do exist. 
Let $B=\left(\begin{array}{rr}1 & 1 \\ 0 & -1\end{array}\right)$ and define $k_{p}: \mathbf{R}^{2} \rightarrow \mathbf{R}^{2}$ by $k_{p}^{2} \rightarrow \mathbf{R}^{2}$ by $k_{p}(x, y)=(x+1 / p, y)$. A Morse-Smale diffeomorphism with periodic data $\boldsymbol{P}$ will be obtained by composing $B$ and $k_{p}$ with a map $\varphi$ which is isotopic to the identity.

For $p=1 \varphi$ is chosen to be a diffeomorphism which commutes with $B$, is isotopic to the identity, and has the fixed hyperbolic dynamics on the unit square which are shown in Figure 4. Note that the polygons are preserved by $B$. Thus the periodic data of the map induced by $B \circ \varphi$ is $\{(1,0,+1),(1,2,-1),(2,1,+1)\}$.

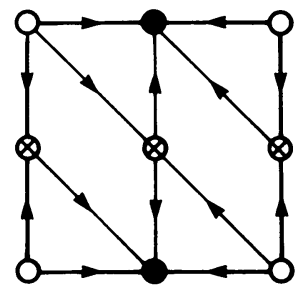

FIGURE $4(p=1)$

We now describe the unit square dynamics of $\varphi$ for the general case. For $i=0, \ldots, 2 p$ the point $(1 / 2, i / 2 p)$ is a hyperbolic fixed saddle. Fixed hyperbolic sources and sinks occur at $(0, i / p)$ and $(0,(2 i+1) / 2 p)$ respectively (see Figure 5$)$.

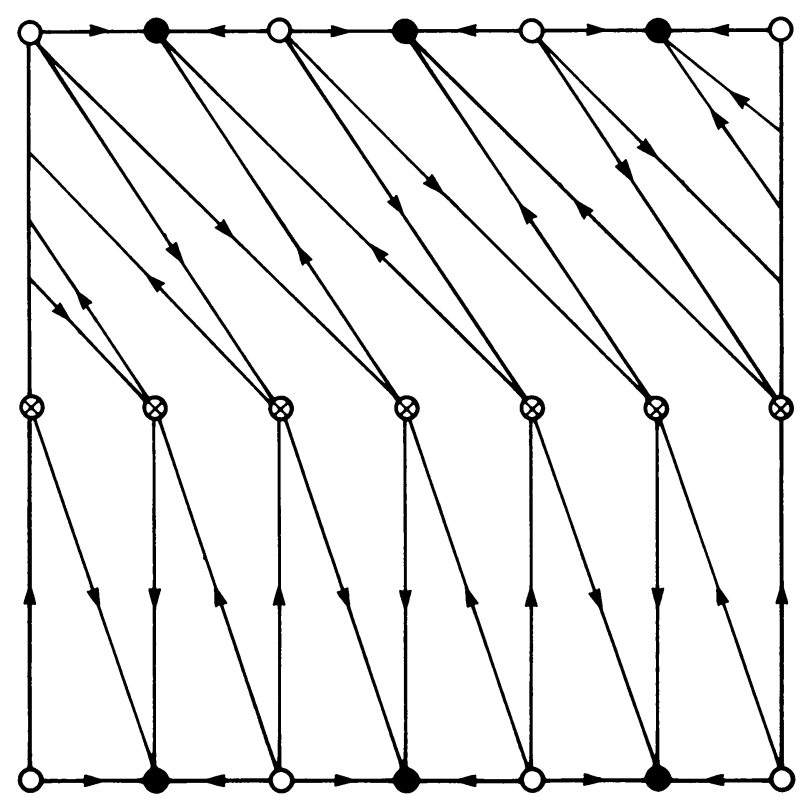

Figure $5 \quad(p=3)$

Consider the segment from $(0,0)$ to $(0,1 / 2)$ and its period $2 p$ orbit in $T^{2}$ under $B \circ k_{p}$. The even iterates are vertical lines from the bottom sources to the saddles. Odd iterates are parallel lines from the top sources to the saddles. Each of these lines will be half of the stable manifold for its saddle endpoint. Following a similar 
procedure with the line from $(1,0)$ to $((2 p-1) / 2 p, 1 / 2)$ generates the other half of the stable manifold (subject to smoothing). The unstable manifolds are obtained by iterating the lines from $(0,1 / 2)$ to $(1 / 2 p, 0)$ and $(1 / 2 p, 1 / 2)$ to $(1 / 2 p, 0)$.

Perturbing near the saddles yields smooth curves which will be the stable and unstable manifolds of $\varphi$. Now let $\varphi$ be a diffeomorphism with these dynamics which commutes with $B \circ k_{p}$. Then $B \circ k_{p} \circ \varphi$ induces an odd type Morse-Smale diffeomorphism with periodic data $P$.

\section{REFERENCES}

[B] S. Batterson, The dynamics of Morse-Smale diffeomorphisms on the torus, Trans. Amer. Math. Soc. 256(1979), 395-403.

[B-F] P. Blanchard and J. Franks, The dynamical complexity of orientation reversing homeomorphisms of surfaces (preprint).

[F] J. Franks, Some smooth maps with infinitely many hyperbolic periodic points, Trans. Amer. Math. Soc. 226 (1977), 175-179.

[H] M. Handel, The entropy of orientation reversing homeomorphisms of surfaces (preprint).

[M] W. Massey, Algebraic topology: An introduction, Harcourt, Brace and World, New York, 1967.

[N] C. Narasimhan, The periodic behavior of Morse-Smale diffeomorphisms on compact surfaces, Trans. Amer. Math. Soc. 248(1979), 145-169.

[N] Z. Nitecki, Differentiable dynamics, M.I.T. Press, Cambridge, Mass., 1971.

[P] J. Palis, On Morse-Smale dynamical systems, Topology 8(1969), 385-405.

[P-S] J. Palis and S. Smale, Structural stability theorems, Proc. Sympos. Pure Math., vol. 14, Amer. Math. Soc., Providence, R.I., 1970, pp. 223-231.

Department of Mathematics, Emory University, Atlanta, Georgia 30322

Current address: School of Mathematics, Institute for Advanced Study, Princeton, New Jersey 08540 